\title{
Ansiedad y los estilos de afrontamiento en los estudiantes de la carrera de Psicología Educativa
}

\author{
Anxiety and coping styles in educational psychology students
}

Ansiedade e estilos de enfrentamento em estudantes de psicologia educacional
Jhonny Santiago Torres-Peñafiel
jstorres@unach.edu.ec
https://orcid.org/0000-0002-5325-8852

\author{
William Marcelo Guaman Paguay \\ willi4mgu4m4n@gmail.com \\ https://orcid.org/0000-0003-3418-1893
}

Universidad Nacional de Chimborazo, Riobamba-Ecuador

Artículo recibido en junio 2021, revisado en julio 2021, arbitrado en agosto 2021 y publicado en septiembre 2021

\section{RESUMEN}

La investigación tuvo como objetivo determinar la correlación de la ansiedad y los estilos de afrontamiento de los estudiantes de la carrera de Psicología Educativa. Se trata de un estudio con un enfoque de investigación cuantitativo, descriptivo y correlacional, se trabajó con el Inventario de Ansiedad de Beck y un Cuestionario de Afrontamiento del Estrés, el mismo que permitió describir la relación de las variables de la población de estudio, en el cual, se encuesto una muestra de 58 jóvenes estudiantes que cumplieron los criterios del programa SPSS 23 que se utilizó para describir y analizar los datos obtenidos. Como resultados se puede constatar que existe correlación inversamente proporcional entre las dos variables dentro de los resultados obtenidos p-0,38, por la cual se obtiene una correspondencia entre la ansiedad y los estilos de afrontamiento, identificando el lado positivo de los problemas y asimismo aprendiendo de las dificultades que se presentan.

Palabras clave: Ansiedad; Estilos de afrontamiento; Estudiantes; Psicología

\section{ABSTRACT}

The objective of the research was to determine the correlation of anxiety and the coping styles of the students of the Educational Psychology career. This is a study with a quantitative, descriptive and correlational research approach, we worked with the Beck Anxiety Inventory and a Stress Coping Questionnaire, the same one that allowed to describe the relationship of the variables of the study population, in which, a sample of 58 young students who met the criteria of the SPSS 23 program was surveyed, which was used to describe and analyze the data obtained. As results, it can be seen that there is an inversely proportional correlation between the two variables within the results obtained $\mathrm{p}-0.38$, by which a correspondence between anxiety and coping styles is obtained, identifying the positive side of the problems and also learning from the difficulties that arise.

Key Words: Anxiety; Coping styles; Students; Psychology

\section{RESUMO}

O objetivo da pesquisa foi verificar a correlação da ansiedade e os estilos de enfrentamento dos alunos da carreira de Psicologia Educacional. Trata-se de um estudo com abordagem quantitativa, descritiva e correlacional, trabalhamos com o Inventário de Ansiedade de Beck e um Questionário de Enfrentamento do Estresse, o mesmo que permitiu descrever a relação das variáveis da população estudada, na qual, uma amostra de 58 jovens estudantes que atendiam aos critérios do programa SPSS 23, que foi utilizado para descrever e analisar os dados obtidos. Como resultados, pode-se perceber que existe uma correlação inversamente proporcional entre as duas variáveis dentro dos resultados obtidos p-0,38, pelos quais se obtém uma correspondência entre ansiedade e estilos de enfrentamento, identificando o lado positivo dos problemas e também aprendendo com as dificuldades que surgem.

Palavras-chave: Ansiedade; Estilos de enfrentamento; Estudantes; Psicologia 


\section{INTRODUCCIÓN}

La ansiedad se define como el conjunto de reacciones corporales que dispone al cuerpo para la acción, en otras palabras, es una reacción necesaria para llevar a cabo respuestas cognitivas y conductuales de actividades cotidianas y especiales, las cuales, son vinculadas con la sobrevivencia (Mercedes, 2016).

Martín (2016) expresa que los estilos de afrontamiento forman parte de los recursos psicológicos de los individuos y es una de las variables personales como participantes en los niveles de calidad de vida, a la cual se atribuye una importancia sobre el bienestar psicológico.

Esta investigación se concreta dentro del contexto educativo superior que se vive en el día a día. La ansiedad y los estilos de afrontamiento son temáticas estudiadas por la ciencia de la Psicología, pues las preocupaciones son un sistema de alerta. $\mathrm{La}$ activación ante estas situaciones son las formas como actuamos ante las mismas en el medio social, laboral $\mathrm{o}$ académico.

Para conocer el alcance de este problema, se estudian los efectos fisiológicos y psicológicos que no sólo perjudican al ser humano que vive su situación, sino también a los que se encuentran a su alrededor. Por esta razón, se toman en cuenta aquellas situaciones que podrían ser las promotoras de un desequilibrio en el individuo.

Cabe indicar que la forma que utilice el individuo para solucionar el problema y esta se caracterice por tener una ejecución no solo de tipo cognitiva, puesto que suele venir acompañada de conductas, está influenciada por una serie de elementos de tipo personal y ambiental, lo que hace que exista una singularidad de resolución, la misma que en ocasiones será eficaz de forma indefinida o funcionaria como un atenuante.
En consecuencia, afirma Fernández (2015) que la ansiedad es una parte fundamental en las experiencias emocionales de las personas, con ella se transmite información basándose en una serie de respuestas que abarca aspectos cognitivos de tensión; aspectos orgánicos, simbolizados por un nivel elevado del sistema nervioso autónomo, y aspectos motores que se adhieren en nuestro contexto que posee dominio en los ejercicios que podemos llevar a cabo, generando acciones inseguras e inestables.

Según Fabra (2018) en la ansiedad lo primordial es el equilibrio que se debe mantener entre el temperamento y carácter, ya que estos dos engloban la personalidad de los jóvenes. Asimismo, existen acciones o funciones psicológicas como pensar, sentir, intuir y percibir que en cada persona es diferente, un ejemplo práctico se presenta cuando alguien es impulsivo. Esto se debe a que prevalecen las funciones de intuir y percibir, precedentemente que las de sentir y pensar.

Por su parte, López (2019) manifiesta que la ansiedad es una emoción que todo el mundo ha experimentado en algún momento y que ayuda al organismo a prepararse para hacer alguna cosa importante. La ansiedad produce una reacción psicofisiológica de activación intensa del sistema nervioso central y de todo el organismo. Aparece cuando se ha de actuar en una situación que demanda un esfuerzo intenso o sostenido y sirve para activar y hacer frente a una amenaza o peligro que está ocurriendo en el presente o que puede ocurrir en el futuro.

En cuanto al afrontamiento, al ser un factor positivo, debe ser un protector de la salud y al ser negativo puede ser un componente de riesgo para enfermarse. El afrontamiento sostiene la ventaja de un cierto control de la situación que puede fluctuar desde el dominio del control de solo las emociones suscitadas por el evento estresante. 
Según Arcas (2015) se entiende que los estilos y estrategias logran ser puestos en marcha ante muchas costumbres y vivencias que no tienen por qué ser vistas propiamente como estresantes, sino como formas en que la persona hace frente a situaciones o inconvenientes, donde se necesitan movimientos de esfuerzos tanto cognitivos como comportamentales en el intento de disminuir o eliminar la experiencia de ansiedad.

Lo anterior es propicio para el desarrollo del presente estudio, porque, a partir de lo expresado por González (2013), dentro del sistema educativo se marca la etapa universitaria estableciendo un método, por el cual se prepara y brinda herramientas a la persona para poder iniciar el cambio al nivel superior, donde suele ser uno de los más conflictivos para el individuo, ya que éste va de la mano con cambios físicos y emocionales característicos de la juventud y eso los aturde, el proceso de adaptación a veces es muy fácil para la mayoría pero para otras personas es difícil aceptar que dejarán atrás su adolescencia para convertirse en jóvenes adultos.

La ansiedad y los estilos de afrontamiento es una parte de la vida estudiantil, donde las personas sienten un grado de la misma, siendo ésta una respuesta adaptativa frente a su desempeño académico, por ende, un grado de angustia o inclusive delantera a su imagen en general es, sin duda, una de las problemáticas que acarrean los jóvenes universitarios del séptimo semestre de la carrera de Psicología Educativa de la Universidad Nacional de Chimborazo, Ecuador.

En el séptimo semestre, muchos de los estudiantes presentan dificultades a causa de un factor emocional y social. En el ámbito educativo se ha observado notablemente cómo los estudiantes muestran síntomas de ansiedad al no haber un desahogue por la variable mencionada, que, por medio del estudio de la misma, a través de instrumentos psicométricos, se actuara con medidas para la reducción de la ansiedad, reconociendo los síntomas para poderlos manejar de mejor manera.

Es pertinente acotar que, en la carrera de Psicología Educativa de la Universidad Nacional de Chimborazo, Ecuador, se ha detectado un conjunto de dificultades entre la ansiedad y los estilos de afrontamiento. Dentro de lo manifestado por los estudiantes de la localidad y de manera personal se ha palpado dichas dificultades, las mismas deben ser trabajadas en los ámbitos educativo, emocional y social para fortalecer su homeostasis, porque si se logra elevar su nivel emocional y conductual se podrá obtener cambios significativos en sus relaciones interpersonales e intrapersonal dentro del séptimo semestre, quienes serán los beneficiarios de dicha investigación.

Para atender esta situación, los autores del presente estudio se plantearon como objetivo general determinar la ansiedad y los estilos de afrontamiento de los estudiantes de la carrera de Psicología Educativa.

Los objetivos específicos de este estudio fueron a) establecer los niveles de ansiedad de los estudiantes de la carrera de psicología educativa de la UNACH, el Inventario de Ansiedad de Beck, por medio del análisis factorial exploratorio y comprobar su eficacia convergente por medio del instrumento que más se utiliza para medir la ansiedad en personas jóvenes adultas; b) identificar los estilos de afrontamiento de los estudiantes, el Cuestionario de Afrontamiento del Estrés que es una medida de autoinforme diseñada para evaluar siete estilos básicos de afrontamiento y c) analizar la correlación de la ansiedad con los estilos de afrontamiento de los estudiantes.

En este contexto tanto social como educativo, el trabajo de investigación se centró en estudiar el efecto de la correlación entre la ansiedad y 
los estilos de afrontamiento sobre el margen de la búsqueda de información, la observación en el comportamiento de los estudiantes sobre la problemática y la aplicación de técnicas de investigación e instrumentos de recolección de datos y su análisis para establecer una relación, conclusiones y recomendaciones que posibiliten respuestas a las diferentes hipótesis y preguntas de investigación, además a la resolución del problema, apoyados de la total colaboración de los estudiantes para indagar dicha problemática de investigación.

\section{MÉTODO}

El trabajo de investigación se desarrolló en un periodo comprendido entre abril-Agosto 2019. En ese lapso se observaron las diferentes variables por singularidad y correlación al comportamiento de varios estudiantes, con un enfoque cuantitativo que se basó en los objetivos de investigación establecidos, además, en lo que corresponde a: recolección de datos, tabulación, análisis e interpretación de estos estudios muéstrales representativos. El diseño de la investigación fue no experimental, porque su carácter socio-educativo, dado que, la ansiedad y los estilos de afrontamiento pertenecen al área de conocimiento de las ciencias de la educación (Psicología Educativa), es decir, cuando el estudiante tiene altos niveles de ansiedad presenta distintas formas de afrontamiento a las circunstancias diarias que se presentan. Asimismo, este estudio fue básico, ya que se obtuvo y recopiló la información para ir construyendo una base de conocimiento que se va agregando a la información previa existente.

Se realizó en el lugar de los hechos, recolectando datos e información directamente de la realidad de la carrera de Psicología Educativa de la Universidad Nacional de Chimborazo (UNACH), Ecuador. Los participantes fueron 58 estudiantes de la carrera de Psicología Educativa. De la muestra total se presenta el $39,7 \%$ de un nivel moderado de ansiedad y un 39,7\% de Estilo de afrontamiento de Reevaluación positiva (Tabla 1).

Tabla 1. Técnicas e instrumento de la investigación.

\begin{tabular}{|c|c|c|c|}
\hline Variable & Técnica & Instrumento & Característica \\
\hline Ansiedad & $\begin{array}{c}\text { Test } \\
\text { Psicométrico }\end{array}$ & $\begin{array}{c}\text { Inventario de } \\
\text { Ansiedad de Beck }\end{array}$ & $\begin{array}{l}\text { Es un cuestionario autoadministrado que consta de } 21 \text { preguntas } \\
\text { de respuesta múltiple. Está compuesto por ítems relacionados con } \\
\text { síntomas depresivos, como la desesperanza e irritabilidad, cogniciones } \\
\text { como culpa o síntomas físicos relacionados con la depresión, por } \\
\text { ejemplo: la fatiga, pérdida de peso y de apetito sexual. }\end{array}$ \\
\hline $\begin{array}{c}\text { Estilos de } \\
\text { afrontamiento }\end{array}$ & $\begin{array}{c}\text { Test } \\
\text { Psicométrico }\end{array}$ & $\begin{array}{l}\text { Cuestionario de } \\
\text { Afrontamiento } \\
\text { del Estrés }\end{array}$ & $\begin{array}{l}\text { El Cuestionario de Afrontamiento del Estrés (CAE) es una medida } \\
\text { de autoinforme diseñada para evaluar siete estilos básicos de } \\
\text { afrontamiento: (1) focalizado en la solución del problema, (2) } \\
\text { autofocalización negativa, (3) reevaluación positiva, (4) expresión } \\
\text { emocional abierta, (5) evitación, (6) búsqueda de apoyo social, y (7) } \\
\text { religión. }\end{array}$ \\
\hline
\end{tabular}

Fuente: Universidad Nacional de Chimborazo, Ecuador 
RESULTADOS Y DISCUSIÓN

Luego de realizar el análisis e interpretación de datos, en relación a las dimensiones e indicadores de los instrumentos de recolección de datos aplicados se establecen los siguientes resultados:

En la ansiedad se puede evidenciar que la gran mayoría de los estudiantes de séptimo semestre se encuentran en un nivel moderado de ansiedad, lo cual se manifiesta en la preocupación de los estudiantes ante el miedo y la inseguridad para seguir adelante, asimismo, en los estilos de afrontamiento podemos evidenciar que la gran mayoría de los estudiantes de séptimo semestre demuestra un estilo de afrontamiento de Reevaluación positiva, indicándonos que los estudiantes saben reconocer y aceptar los diferentes problemas cotidianos. Y, por ende, en la correlación se puede constatar que existe correlación inversamente proporcional entre las dos variables dentro de los resultados obtenidos p- 0,38 , por la cual se obtiene una correspondencia entre la ansiedad y los estilos de afrontamiento, identificando el lado positivo de los problemas y asimismo aprendiendo de las dificultades que se presentan.

En cuanto al nivel de ansiedad, los sujetos obtuvieron una frecuencia clasificada en Leve (18); moderado (23) y grave (10). (Tabla 2).

Tabla 2. Nivel de ansiedad.

\begin{tabular}{llcccc}
\hline & Frecuencia & Porcentaje & Porcentaje válido & Porcentaje acumulado \\
\hline Válido & Mínimo & 7 & 9,5 & 12,1 & 12,1 \\
& Leve & 18 & 24,3 & 31,0 & 43,1 \\
& Moderado & 23 & 31,1 & 39,7 & 17,2 \\
\\
Grave & 10 & 13,5 & $\mathbf{1 0 0 , 0}$ & 100,0 \\
\hline
\end{tabular}

Fuente: Inventario de Ansiedad de Beck.

Con respecto a los estilos de afrontamiento ANÁLISIS se pudo mostrar que se ha podido evidenciar que de los 58 estudiantes, 14 presenta el 24,1 manifestando un estilo de afrontamiento de Focalizado en la solución de problemas, 3 presentan el 3,2\% manifestando un la Autofocalización negativa, 23 presentan el 39,7\% manifestando la Reevaluación positiva, 6 presentan el 10,3\% manifestando la Expresión emocional abierta, 3 presentan el 5,2\% manifestando la Evitación, 5 presentan el 8,6\% revelando la Búsqueda de apoyo social, 4 presentan el 6,9\% manifestando la Religión. (Tabla 3). 
Tabla 3. Estilos de afrontamiento.

\begin{tabular}{llrrrr}
\hline & Frecuencia & Porcentaje & $\begin{array}{c}\text { Porcentaje } \\
\text { válido }\end{array}$ & $\begin{array}{c}\text { Porcentaje } \\
\text { acumulado }\end{array}$ \\
\hline Válido & Focalizado en la solución de problemas & 14 & 18,9 & 24,1 & 24,1 \\
& autofocalización negativa & 3 & 4,1 & 5,2 & 29,3 \\
& Reevaluación positiva & 23 & 31,1 & 39,7 & 69,0 \\
Expresión emocional abierta & 6 & 8,1 & 10,3 & 79,3 \\
Evitación & 3 & 4,1 & 5,2 & 84,5 \\
Búsqueda de apoyo social & 5 & 6,8 & 8,6 & 93,1 \\
Religion & 4 & 5,4 & 6,9 & 100,0 \\
Total & $\mathbf{5 8}$ & $\mathbf{7 8 , 4}$ & $\mathbf{1 0 0 , 0}$ & \\
\hline
\end{tabular}

Fuente: Cuestionario de Afrontamiento del estrés.

Análisis de la correlación de las variables

Entre las variables Ansiedad y Estilos de aprendizaje se pudo evidenciar de los 58 estudiantes,
9 presenta el $12 \%$ manifestando un nivel alto, 27 presenta el $34 \%$ un nivel medio, 42 expresa el $54 \%$ un nivel bajo. (Tabla 4 y Gráfico 1).

Tabla 4. Correlación de las variables ansiedad - estilos de afrontamiento.

\begin{tabular}{llrr}
\hline & & Nivel de ansiedad & Estilo de Afrontamiento \\
\hline Nivel de ansiedad & Correlación de Pearson & 1 &, 038 \\
& Sig. (bilateral) & &, 777 \\
& $\mathrm{~N}$ & 58 & 58 \\
Estilos de Afrontamiento & Correlación de Pearson &, 038 & 1 \\
& Sig. (bilateral) &, 777 & 58 \\
\hline
\end{tabular}

Fuente: Inventario de Ansiedad de Beck - Cuestionario de Afrontamiento del estrés. 


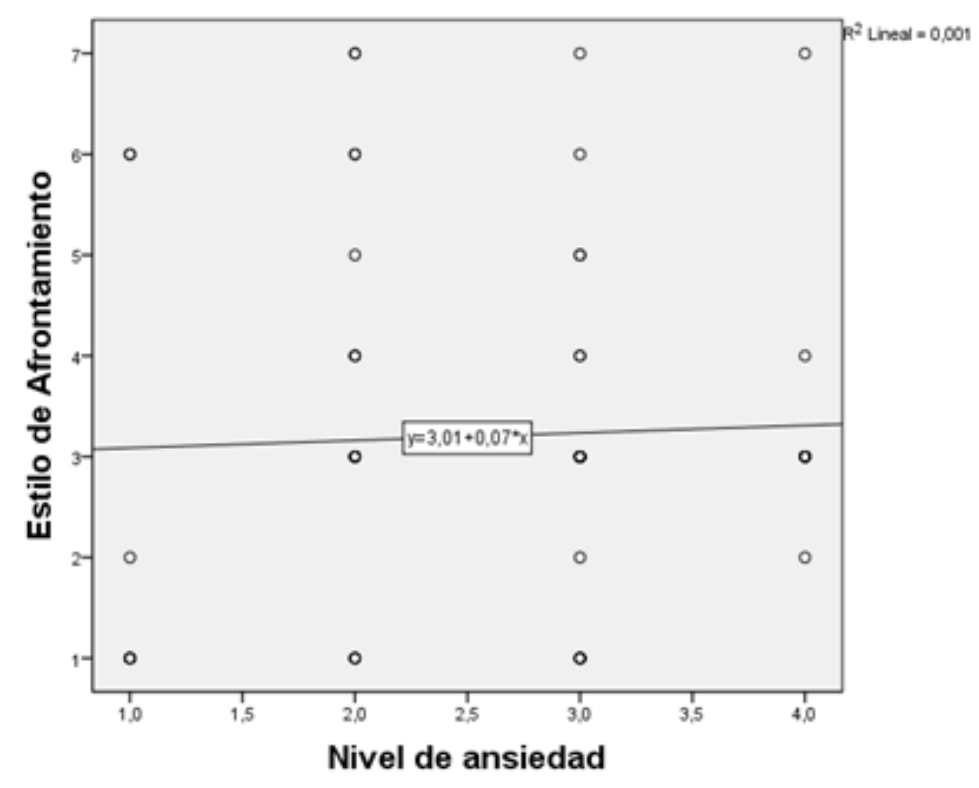

Gráfico 1. Correlación de las variables Ansiedad-Estilos de Afrontamiento. (Fuente: Inventario de Ansiedad de Beck Cuestionario de Afrontamiento del estrés).

\section{Discusión}

Aunque en la actualidad las investigaciones se aseguran que el afrontamiento hace referencia a las distintas formas con las que las personas se enfrentan a situaciones estresantes. Las investigaciones señalan que, a elevada ansiedad, mayor uso de estrategias de afrontamiento adaptativas y menor uso de desadaptativas.

$\mathrm{Al}$ analizar factorialmente la ansiedad se obtuvieron cuatro niveles. No obstante, basándose en la puntuación directa se pudo evidenciar que la gran mayoría de los estudiantes se encuentran en un nivel moderado de ansiedad con un $39,7 \%$, lo cual se manifiesta en la preocupación de los estudiantes ante el miedo y la inseguridad para seguir adelante, además demuestra un desequilibrio en sus pensamientos perjudicando su concentración. Los otros tres niveles presentan el 12,1\% manifestando un nivel mínimo, el 31,0\% un nivel leve, y el 17,2 un nivel grave.

En las investigaciones anteriores el estrés es considerado como un estado producido por una serie de demandas inusuales o excesivas que amenazan con el bienestar o integridad de una persona, asimismo, las relaciones entre los estilos de afrontamiento son, en general, ponderadas, lo que apoya un planteamiento multidimensional del afrontamiento. Curiosamente, como señala, los diferentes estilos ayudan a una mejor resolución de problemas, que de los siete estilos de afrontamiento se evidencia que, de los 58 estudiantes, el 24,1 manifestando un estilo de afrontamiento de Focalizado en la solución de problemas, 3 el 3,2\% manifestando un la Autofocalización negativa, 23 presentan el 39,7\% manifestando la Reevaluación positiva, el 10,3\% manifestando la Expresión emocional abierta, el 5,2\% manifestando la Evitación, el 8,6\% manifestando la Búsqueda de apoyo social, el 6,9\% manifestando la Religión, esto quiere decir que la gran mayoría de los estudiantes de séptimo semestre demuestra un estilo de afrontamiento de Reevaluación positiva, indicando que los estudiantes saben reconocer y aceptar los diferentes problemas cotidianos, identificando 
el lado bueno y malo de las distintas situaciones aprendiendo de las mismas.

La correlación, entre la ansiedad y los estilos de afrontamiento se sitúan en un valor humano, lo cual represente un estudio con una clara relación en los estudiantes de séptimo semestre de la carrera de Psicología Educativa de la UNACH con situaciones sociales.

Aunque, en ambos casos, la relación de la ansiedad con los estilos de afrontamiento, considerados globalmente, es moderada (negativa en el caso de la ansiedad y positiva en el caso de los estilos de afrontamiento), no parece ocurrir lo mismo a nivel cualitativo, estas diferencias entre estas dos variables se mantienen a la hora de evaluar comportamientos manifestados de los objetos de la investigación.

Al centrar la atención en las relaciones entre las dimensiones de la ansiedad y los estilos de afrontamiento, los resultados son inversamente proporcional a las mismas. Las extensiones de la ansiedad tienen que ver con el ámbito social, ya que afecta al equilibrio emocional que debe mantener una persona implicando factores cognitivos, fisiológicos y conductuales o motores, asimismo, las diferentes dimensiones de la segunda variable se concretan en un conjunto de habilidades cognitivas y conductuales que el individuo maneja para precisar situaciones internas o externas que sean percibidas como excesivas para los recursos de la persona.

La correlación entre la Ansiedad y los Estilos de Afrontamiento se asegura como un constructo de la investigación, donde se evidencia que, de los 58 estudiantes, el 12\% manifestando un nivel alto, el $34 \%$ un nivel medio, el 54\% un nivel bajo. Esto explica porque dentro de los resultados que se obtiene en el programa SPSS 23 y tomando en cuenta que el p-valor base es de 0,05 , se puede constatar que existe una correlación inversamente proporcional entre las dos variables dentro de los resultados obtenidos $\mathrm{p}-0,38$, por la cual se obtiene una correspondencia entre la ansiedad y los estilos de afrontamiento, identificando el lado positivo de los problemas y asimismo aprendiendo de las dificultades que se presentan.

Esta situación correlacional es significativa entre dichas variables de estudio no se alejan mucho de la realidad, especialmente cuando se hace referencia a los factores que influyen en el estudio, ya que los estudiantes de séptimo semestre de la carrera de Psicología Educativa muestran inquietudes, inseguridades para trascender, pero al mismo tiempo reconocen y aceptan las distintas dificultades equilibrando la parte correcta e incorrecta de las múltiples situaciones aprendiendo de las mismas.

\section{CONCLUSIONES}

Se establece que existen niveles relevantes de ansiedad moderada, manifestando vacilaciones como la desconfianza y un desequilibrio que detiene al individuo para trascender, no tienen seguridad en sí mismos, pero esto sin duda puede variar con relación a los contextos en los que pueden estar desarrollando y llegar a tener una satisfacción ante los problemas.

Se identifica que generalmente los estudiantes manifiestan un estilo de afrontamiento de Reevaluación positiva, que con la misma permite modificar conductas sin distorsionar la realidad, reconociendo, las diferentes situaciones problemáticas para llegar a su autorrealización, fijándose de una forma relativa.

Se analiza que existe una correlación inversamente proporcional muy significativa entre la ansiedad con los estilos de afrontamiento de los estudiantes de séptimo semestre de la carrera 
de Psicología Educativa de la UNACH, dado que presentan un nivel relevante de ansiedad, demostrando una sintomatología de inestabilidad emocional y falta de homeostasis, afectando el desarrollo autónomo.

REFERENCIAS

Arcas, M. (16 de 06 de 2015). Afrontamiento y salud mental en el ámbito de la formación universitaria de enfermería. Universidad de los andes. Mérida-Venezuela. https://repositorio. uam.es/bitstream/handle/10486/10930/54521_ T E S I S \% 20 M O R A I M A \% 20 P E N A. pdf? sequence $=1 \&$ isAllowed $=y$

Fabra, A. (2018). Revista Complutense de Educación. Disponible en: file://C:/Users/ USER/Downloads/17441-Texto\%20del\%20 art\%C3\%ADculo-17517-1-10-20110602\%20 (1).PDF
Fernández, A. (2015). Revista Educacion. Disponible en: https://www.redalyc.org/ pdf/440/44012058011.pdf

González, F. (2013). Estrategias de enseñanza: investigaciones sobre didáctica en instituciones. Disponible en http://biblioteca.clacso.edu.ar/ Colombia/fce-

López, E. (2019). Clinic Barcelona. Obtenido de https://www.clinicbarcelona.org/asistencia/ enfermedades/trastornos-de-ansiedad/ definicion

Martín., Y. D. (14 de 06 de 2016). Scielo. Disponible en: http://scielo.sld.cu/pdf/hmc/v10n1/ hmc070110.pdf

Mercedes, L. d. (21 de 01 de 2016). Disponible en: https://repositorio.uta.edu.ec/ bitstream/123456789/18011/2/LUCIA\%20 VACA\%20-\%20ESTRES\%20Y\%20TIPOS\%20 DE \% 20 A F RONTAMIEN TO \% 20 AL \% 20 ESTRES.pdf 